Anuario Latinoamericano Ciencias Políticas

y Relaciones Internacionales

vol. 1, 2014

p. $127-141$

\title{
Medidas de Fomento de la Confianza y Seguridad en el ámbito de la Organización de Estados Americanos
}

\author{
Pawet Trefler \\ PAŃSTWOWA WYŻSZA SZKOŁA WSCHODNIOEUROPEJSKA \\ EN PRZEMYŚL, POLONIA \\ $\triangle$ pawel.trefler@pwsw.eu
}

\begin{abstract}
RESUMEN
El presente artículo trata sobre el proceso de construcción del régimen de Medidas de Fomento de la Confianza y Seguridad (MFCS) en el ámbito de la Organización de Estados Americanos. Se ha realizado un análisis del concepto de las MFCS, el cual se ha venido desarrollando en el foro interamericano desde el principio de los años 90. Se han presentado problemas respecto a la falta de involucramiento de algunos países de América Latina en la implementación de las MFCS, a la vez llamando la atención sobre el hecho de que en esta región se las utiliza, principalmente, en las relaciones bilaterales. Además, se ha hecho la evaluación del estado de las MFCS en el hemisferio occidental y se ha presentado las perspectivas de su futuro desarrollo, propagación y validación de su uso en América del Sur.
\end{abstract}

PALABRAS CLAVE: seguridad, medidas de fomento de la confianza, Organización de Estados Americanos, América Latina, Estados Unidos.

\section{SUMMARY}

The paper deals with the process of establishing Confidence and Security - Building Measures (CSBMs) under the auspices of the Organization of American States. Analyses are made of the CSBM concept which has been developed since the beginning of the 1990s through Inter-American dialogue. It lays out the problems bound up with a lack of commitment to the implementation of CSBMs seen in some Latin American states, also drawing attention to the fact that in this region they are set in place mainly in bilateral relations. Evaluations of the status of CSBMs in the Western Hemisphere are also made, stating the outlook for their further development, their wider adoption, and the validation of their uses in South America.

KEYWORDS: security, confidence-building measures, Organization of American States, Latin America, United States of America. 


\section{Introducción}

América Latina es generalmente considerada como la región cuyo problema principal no son los conflictos internacionales, sino la inestabilidad interna de los Estados. Por esta razón, en los últimos años, se ha dedicado mucha atención a las cuestiones de la seguridad pública en el contexto de la lucha contra las llamadas "nuevas amenazas" a la seguridad. La prioridad que se atribuye a la cuestión de la construcción de Estados democráticos fuertes, cuyas autoridades son capaces de garantizar la seguridad pública y el imperio de la ley en todo el territorio, es absolutamente racional. En América Latina, la seguridad internacional es la resultante del nivel de seguridad interna de cada país. Parece que, actualmente, el factor de riesgo más grave en las relaciones internacionales en el hemisferio occidental no son los conflictos fronterizos, sino la transferencia de inestabilidad (Arévalo de León 2002: 9). Las organizaciones revolucionarias y grupos criminales organizados que operan en áreas periféricas poco controladas por las autoridades estatales pueden llevar a unas tensiones serias en las relaciones internacionales, tales como, por ejemplo, la crisis diplomática entre Ecuador, Colombia y Venezuela en 2008. ${ }^{1}$ La transferencia de la inestabilidad puede ser consecuencia de profundas crisis políticas, económicas, alimentarias, una migración incontrolada ${ }^{2}$ o catástrofes naturales. El remedio que puede prevenir que las crisis se conviertan en conflictos militares es el desarrollo de las relaciones diplomáticas, económicas, socioculturales y la cooperación militar a fin de reforzar la confianza mutua entre los países. Este proceso es muy complejo, arduo y depende de varios factores estrechamente entrelazados, tanto en la política interior de los países como en el ámbito internacional. El desarrollo de la cooperación internacional debe resultar de la buena voluntad de las partes y basarse en la intención de construir una jerarquía de valores en común para facilitar la comunicación mutua. Además, lo importante es seguir siendo realistas. Por un lado, se trata de darse cuenta de la inutilidad de copiar las soluciones utilizadas en otros lugares y distintas realidades y, por el otro, de reconocer el carácter evolucionario del proceso de construcción de la confianza mutua.

1 Esta crisis tuvo lugar en los días 1-7 de marzo de 2008. Su causa directa fue la incursión de las fuerzas armadas y policía colombiana en el territorio de Ecuador y el asesinato de varios miembros de las Fuerzas Armadas Revolucionarias de Colombia (FARC), además de 5 ciudadanos de México y Ecuador. Una de las personas asesinadas fue Raúl Reyes - un dirigente de alto rango de las FARC. En respuesta a ello, el presidente de Ecuador, Rafael Vicente Correa Delgado, retiró a su embajador en Bogotá y envió las fuerzas militares a la frontera con Colombia. El presidente de Venezuela, Hugo Rafael Chávez Frías, hizo lo mismo, declarando que las acciones de Colombia podían llevar a una guerra. Finalmente, gracias a la mediación del presidente de la República Dominicana, Leonel Antonio Fernández Reyna, durante la cumbre del Grupo de Río en Santo Domingo, el presidente de Ecuador y el presidente de Colombia, Álvaro Uribe Vélez, se dieron un apretón de manos para mostrar simbólicamente el fin del conflicto.

2 Por ejemplo, la llamada "Guerra del fútbol" entre el Salvador y Honduras que tuvo lugar en los días 14-18 de julio de 1969. 
El objetivo de este artículo es mostrar el proceso de construcción y el estado actual del régimen interamericano de medidas de fomento de la confianza y seguridad (MFCS) en el marco de la Organización de Estados Americanos (OEA). Este es un tema de vital importancia, teniendo en cuenta el hecho de que esta organización constituye el foro de consultas internacionales del más alto nivel en todo el hemisferio occidental, marcando las pautas de la política de seguridad en esta parte del mundo. Así pues, en las conferencias organizadas bajo los auspicios de la OEA en Santiago de Chile en los días 8-10 de noviembre de 1995 (Conferencia Regional sobre MFCS), en San Salvador en los días 25-28 de febrero de 1998 (II Conferencia Regional sobre MFCS), en Miami en los días 2-4 de febrero de 2003 (Reunión de Expertos Sobre MFCS) y, luego, durante cinco siguientes Foros sobre MFCS (en 2005, 2006, 2008, 2010 y 2013) fue elaborada la lista consolidada de MFCS (OEA 2009), la cual abarcó en su ámbito un grupo de países muy amplio. Sin embargo, la OEA no dispone de la posibilidad de implementar y evaluar eficazmente el uso de las MFCS en el territorio tan extenso. Por eso es tan importante la cooperación bilateral de los Estados y organizaciones subregionales, las cuales, declarando la voluntad de desarrollar su propio conjunto de las MFCS, pueden referirse a lo que elaboró la OEA. Tal como lo señaló Cristián Garay, las MFCS “son más bien productos residuales de los acuerdos de los ministros de Defensa en reuniones de tipo hemisférico... Lo importante es que, si bien los acuerdos son de carácter hemisférico, sin embargo, su aplicación es de carácter regional..." (Citado por Riquelme Rivera 2011:33).

El artículo se divide en dos partes principales. La primera tiene el carácter teórico y se refiere al origen y esencia del concepto de las MFCS. La segunda presenta el régimen de las MFCS que ha sido creado y funciona en el marco de la OEA, evidenciando sus defectos y presentando las perspectivas de su futuro desarrollo en América Latina.

\section{La génesis y la esencia del concepto de las MFCS}

Se asume que el origen del concepto de las MFCS se remonta al principio de los años 60 del siglo pasado. Después de la crisis cubana, por causa de la cual el mundo se encontró al borde de la tercera guerra mundial, en las relaciones de la Guerra Fría entre el Bloque Occidental y Oriental tuvo lugar una distensión (détente). Se emprendieron entonces los primeros intentos de construcción de confianza entre la Organización del Tratado del Atlántico Norte (OTAN) y el Pacto de Varsovia. Su manifestación material fue el establecimiento, el 30 de agosto de 1963, de una línea de comunicación directa (teléfono rojo) entre la Casa Blanca y el Kremlin. Luego, el 26 de mayo de 1972, EEUU y la URSS firmaron el acuerdo de limitación de armas estratégicas SALT I (Strategic Arms Limitation Treaty). El 1 de agosto de 1975, después de unos años de negociaciones, los representantes de los países europeos neutrales, de la OTAN y del
Medidas de Fomento de la Confianza y Seguridad en el ámbito de la Organización de Estados Americanos

Paweł Trefler 
Pacto de Varsovia adoptaron en Helsinki el Acta Final de la Conferencia sobre la Seguridad y la Cooperación en Europa (CSCE), cuya parte constituyó el Documento sobre las medidas destinadas a fomentar la confianza y sobre determinados aspectos de la seguridad y el desarme. Fueron presentadas entonces las primeras Medidas de Confianza Mutua - $\mathrm{MCM}^{3}$ (Tulliu, Schmalberger 2003: 162), aunque hay que señalar que su importancia en aquel tiempo fue marginal (Lachowski 2004: 10), y, después de la intervención soviética en Afganistán en 1979, las relaciones entre Moscú y Washington se enfriaron significativamente, lo que frenó por varios años el progreso en la construcción del régimen europeo de las MFCS.

El proceso de ampliación y perfeccionamiento de las MCM (su conversión en MFCS) en el marco de la CSCE/OSCE fue arduo y se desarrolló por etapas marcadas por los documentos finales adoptados en las consecutivas conferencias de la CSCE/OSCE (entre otros, el Documento de Estocolmo del 19 de septiembre de 1986, el Tratado de las Fuerzas Armadas Convencionales en Europa (FACE/CFE) del 19 de noviembre de 1990, el Tratado de Cielos Abiertos del 24 de marzo de 1992 y cuatro Documentos de Viena (del 17 de noviembre de 1990, del 28 de noviembre de 1994, del 16 de noviembre de 1999 y del 30 de noviembre de 2011), las cuales introdujeron las nuevas generaciones de las MFCS. Desde 1990, la evaluación de implementación de las MFCS utilizadas por los países de la OSCE se hace durante Annual Implementation Assessment Meeting (AIAM).

En el foro de la Organización de Naciones Unidas (ONU), se ha señalado repetidamente la necesidad de construir la confianza entre los países por medio de las MFCS 4 . Esto ha dado impulso a los países del Oriente Próximo, Asia Sudoriental, América y África para empezar la creación de sus propios regímenes de las MFCS, copiados de las soluciones elaboradas por la CSCE/OSCE.

Sin embargo, no existe una definición única de las MFCS porque se las puede interpretar de dos maneras - en el sentido "estrecho" o "amplio". Según el clásico enfoque "estrecho", las MFCS son "mecanismos técnicos destinados a evitar el estallido de un conflicto" (Caro 1994: 18) a causa de "un accidente, malentendido o una interpretación errónea de las intenciones de un potencial adversario" (Child 1994: 43) o, poniéndolo de otra forma, "disposiciones militares adoptadas por los Estados para disipar la desconfianza que podría llevar a un conflicto armado" (Tulliu, Schmalberger 2003: 159). Claudio A. Fuentes y Paz Millet las definen como "acciones concertadas entre determinados Estados a fin de reducir la probabilidad de conflictos militares, generar conocimiento y confianza con el propósito final de aminorar las posibilidades de un

3 En la llamada "Canasta I" del Acta se establece que las partes deben notificar por anticipado cualquier ejercicio militar que involucre a más de 25.000 efectivos y el intercambio voluntario de observadores militares.

4 Por ejemplo, en el Documento Final del décimo periodo extraordinario de sesiones de la Asamblea General de la ONU dedicado al desarme, Resolución S-10/2 de la AG ONU de 30.06.1978. 
accidente, un malentendido o una interpretación errónea de las intenciones de un potencial adversario" (Fuentes, P. Millet 1997: 19). El enfoque más extenso fue utilizado por Francisco Rojas Aravena - uno de los más conocidos teóricos y prácticos latinoamericanos ${ }^{5}$ en cuanto a la implementación y evaluación de las MFCS. Según él, las MFCS son "acciones bilaterales y multilaterales destinadas a prevenir situaciones de crisis y de conflicto. Buscan fortalecer la paz y la seguridad internacional. Crean una atmósfera propicia para establecer un marco de entendimiento que mitigue las percepciones de amenaza inmediata y eviten eventuales elementos de sorpresa" (Aravena 1996: 43). Mientras tanto, John Holum - el ex director de U.S. Arms Control and Disarmament Agency (ACDA) - afirmó que "Las MFCS obran continuamente para reducir o eliminar las causas de desconfianza, temor, tensiones y hostilidades. Ellas aumentan la franqueza y transparencia respecto a actividades y fuerzas militares y, por lo tanto, permiten que el comportamiento de otros estados sea más predecible y demuestre la ausencia de intenciones hostiles" (Holum 1996: 16).

Las MFCS incluyen los acuerdos sobre control y limitación de armas, el desarme y las MCM militares, las cuales podemos dividir en tres categorías: 1. medidas de información y comunicación; 2. medidas de observación e inspección; 3. restricciones militares (Tulliu, Schmalberger 2003: 159). En la perspectiva muy amplia, aparte de los elementos mencionados más arriba, a las MFCS se incluyen también, entre otros, las medidas que estimulan la cooperación internacional en el ámbito político, económico, sociocultural, humanitario, ecológico, las iniciativas conjuntas en favor de la democracia, buena gobernanza y reforzamiento del control democrático sobre las fuerzas armadas (Arévalo de León 2002: 8; Aravena 1996: 46). Isaac Caro señala que "En el marco de las nuevas tendencias que caracteriza la política global y regional, las MCM deben aplicarse no sólo a la esfera militar y policial, sino también a otros campos de la vida social, política y económica, con el objetivo de combatir y/o prevenir problemas como el narcotráfico, la corrupción, la expansión de epidemias, el incremento de la delincuencia, la degradación del medio ambiente, la pobreza, el hambre, los efectos de las catástrofes naturales" (Caro 1994: 57). Estos requerimientos fueron cumplidos el 4 de febrero de 2003, al final de la reunión de expertos sobre MFCS organizada por la OEA en Miami, cuando fue adoptado el Consenso de Miami, es decir, la Declaración de los expertos sobre MFCS (OEA 2003). Los expertos dividieron las MFCS en: 1. Medidas diplomáticas y políticas; 2 . Medidas educativas y culturales; 3 . Medidas militares relacionadas con: a) despliegue de fuerzas armadas; b) intercambio de información; c) intercambio personal; d) comunicaciones; e) contactos; f) capacitación y educación; g) verificación. Durante el II Foro sobre MFCS, el cual tuvo lugar el 29 de noviembre de 2006 en Washington, fueron distinguidas las MFCS aplicadas al campo de la defensa $\mathrm{y}$ aquellas relacionadas a las nuevas amenazas.

5 F. Rojas Aravena se encontró inscrito en el Registro de expertos en medidas de fomento de la confianza y de la seguridad de la Organización de Estados Americanos.
Medidas de Fomento de la Confianza y Seguridad en el ámbito de la Organización de Estados Americanos

Paweł Trefler 
El primer grupo incluye: 1. Intercambio de doctrinas de defensa; 2. Ejercicios militares conjuntos; 3. Intercambio de personal militar; 4. Transparencia en las adquisiciones de armas; 4. Limitación de gastos militares; 5. Foros militares como Conferencia de Ministros de Defensa de las Américas (CMDA); 5. Destrucción del arsenal de armas. Los trabajos sobre el régimen de MFCS relacionadas a las nuevas amenazas (Morris 1994: 109-113) deben referirse a la creación de herramientas internacionales para la solución de problemas (las llamadas "nuevas amenazas"), tales como: 1. Tráfico de drogas; 2. Tráfico de armas; 3. Crimen organizado transnacional; 4. Migración ilegal; 5. Explotación ilícita de recursos naturales; 6. Desastres naturales (Jácome 2008: 3-4), las cuales pueden contribuir a la transferencia de la inestabilidad.

Las MFCS constituyen una parte integral del concepto de la seguridad cooperativa, la cual tiene el carácter inclusivo, basándose sobre la cooperación voluntaria, coordinada en el diálogo, y las garantías mutuas de seguridad. De acuerdo con este concepto, el logro de una paz justa y duradera es posible sólo bajo la condición de eliminar el llamado dilema de seguri$\mathrm{dad}^{6} \mathrm{y}$, precisamente, para eso deben servir las MFCS. Sin embargo, merece la pena recordar que este tipo de medidas pueden funcionar eficazmente sólo cuando los Estados expresan una auténtica buena voluntad en cuanto a la construcción conjunta de la seguridad democrática basada en las reglas fundamentales de la ley internacional incluidas en la Declaración sobre los principios de Derecho Internacional referente a las relaciones de amistad y a la cooperación entre los Estados de conformidad con la Carta de las Naciones Unidas 2625(XXV) del 24 de octubre de 1970. Igual de importante para la eficacia de las MFCS es la política interior y, en particular, el funcionamiento del gobierno civil democrático estable, que goza de confianza social, ejerce un control real sobre las fuerzas armadas y domina plenamente sobre todo el territorio del Estado. Las MFCS no sirven para superar problemas de los países débiles institucionalmente, los cuales no resolvieron conflictos fronterizos que existen entre ellos (Arévalo de León 2002: 8-10; Bromley, Perdomo 2005) Estas medidas tienen el valor preventivo, pero no pueden contribuir a terminar los conflictos existentes. Para lograr esto, son necesarios acuerdos bilaterales, la mediación internacional o el arbitraje ante La Corte Internacional de Justicia.

Hay dos métodos básicos de clasificación de las MFCS - generacional y de naturaleza. En el primer caso "se destaca un criterio incremental de las medidas de confianza en donde un periodo cimienta el desarrollo de próximo" (Briones, Álvarez 2008: 19). Esto significa que el proceso de la creación de las MFCS recorre de forma progresiva. Así pues, se habla de las generaciones sucesivas de las MFCS. En el caso del más desarrollado régimen de MFCS que

6 El dilema de seguridad se refiere a la situación en la que "el aumento de la seguridad de una parte puede llevar, a la vez, al debilitamiento de la seguridad de la otra parte y al revés” (Zięba 2008: 21). 
funciona bajo los auspicios de la CSCE/OSCE, usualmente, se distingue de tres a cuatro generaciones de las MFCS.

En caso de la clasificación de naturaleza "se ordenan las categorías de acuerdo al tipo de medida que se aplican: declaratorias, de comportamiento, de restricción, de consulta, de comunicación, de intercambio y de verificación" (Briones, Álvarez 2008: 19). Existen también clasificaciones que toman en cuenta, por ejemplo, el objetivo de las medidas utilizadas. Entre estas clasificaciones podemos señalar la de John Borawski quien distingue las medidas siguientes: 1. Medidas de intercambio de información, que tienen por objetivo aumentar el conocimiento mutuo y entendimiento sobre actividades militares, por ejemplo: a) evaluación de presupuestos militares; b) ubicación y organización de principales unidades y comandos; c) notificación de inci-

\begin{tabular}{|c|c|c|}
\hline Años & Generaciones de medidas & $\begin{array}{l}\text { Nombre de la } \\
\text { generación }\end{array}$ \\
\hline 1975-1986 & $\begin{array}{l}\text { I generación } \\
\text { - notificación y acceso } \\
\text { - inspección voluntaria } \\
\text { MCM comienzan a ser llamadas MFCS }\end{array}$ & voluntaria \\
\hline 1986-1990 & $\begin{array}{l}\text { II generación } \\
\text { - intercambio de información } \\
\text { - observación } \\
\text { - inspección } \\
\text { - intercambio de personal } \\
\text { Incluye medidas militares y obligatorias a nivel } \\
\text { político con formas de verificación obligatorias. }\end{array}$ & obligatoria \\
\hline 1990-1992 & $\begin{array}{l}\text { III generación } \\
\text { - restricciones sobre actividades y material } \\
\text { bélico } \\
\text { - contactos militares más allá de la inspección } \\
\text { - centros de comunicación directa a nivel de } \\
\text { - jefes militares } \\
\text { - acceso amplio más allá de inspecciones }\end{array}$ & restrictiva \\
\hline 1992-2013 & $\begin{array}{l}\text { IV generación } \\
\text { - medidas de intercambio de planificación de } \\
\text { defensa } \\
\text { - ejercicios conjuntos } \\
\text { - principios de transferencia de armas } \\
\text { - medidas para crisis localizadas }\end{array}$ & de intercambio \\
\hline
\end{tabular}

Fuente: S. R. Briones, R. V. Álvarez, ¿Construyendo confianzas? Fronteras, FFAA y Política en América Latina, Santiago de Chile 2008, p. 20.
Medidas de Fomento de la Confianza y Seguridad en el ámbito de la Organización de Estados Americanos

Paweł Trefler

Tabla 1.

Generaciones de MFCS. 
dentes nucleares, no autorizados o inexplicables; d) notificación de maniobras y pruebas de lanzamiento de misiles; e) enlaces de comunicación; 2. Medidas de observación e inspección, cuyo propósito es permitir una evaluación independiente del carácter de las actividades militares y así aliviar o confirmar las sospechas, por ejemplo: a) la presencia de observadores en las principales maniobras; b) inspecciones en sitio; c) instalación de sensores en los silos de misiles balísticos intercontinentales; d) favorecimiento de condiciones para contactos de misiones oficiales militares y personal militar acreditado. 3. Medidas de restricción de las actividades militares, siendo la finalidad de éstas impedir el empleo o despliegue de fuerzas militares en modos potencialmente amenazantes, por ejemplo: a) prohibición de ataques simulados; b) prohibición de futuras instalaciones de armas ofensivas y equipo de apoyo; c) prohibición de lanzamientos múltiples de misiles; d) límites de movimientos y maniobras; e) creación de zonas libres de determinados armamentos (Caro 1994: 39).

Los dos métodos de clasificación de las MFCS tienen fallos fundamentales (Briones, Álvarez 2008: 20-21), en particular, cuando los intentaremos trasladar a las realidades latinoamericanas. Así es, sobre todo, por causa de diferentes premisas y expectativas sobre el papel que las MFCS tenían que cumplir inicialmente en la región euroatlántica y en América Latina. Fernando Bustamante recordó que durante la guerra fría las fuerzas armadas de la OTAN y del Pacto de Varsovia fueron capaces de poner en marcha la destructiva campaña militar en muy poco tiempo, así pues, al principio, el objetivo principal de las MFCS fue de "evitar que la guerra comenzara debido a un malentendido respecto a intenciones del bando opuesto" (Bustamante 1996: 204). Por eso, estas medidas tenían el "carácter exclusivamente militar-convencional y estaban pensadas como una serie de mecanismos que redujeran la probabilidad de que los respectivos Estados mayores pudiesen cometer errores de cálculo respecto a las intenciones del contrario" (Ibidem). Vale la pena señalar que estas medidas "no requerían de las partes a) modificaciones de su sistema político y régimen estatal; b) no requerían de garantías con respecto a la estabilidad interna de las partes, ni dependían de ella; c) sí requirieron eventualmente de un proceso concomitante de reconocimiento de fronteras y de la integridad territorial de las partes" (Ibidem). Requerían también el "desarrollo de una conciencia común de interés compartido en la paz, o al menos en la no guerra" y además, "no era una condición previa necesaria el desarrollo de una fuerte interdependencia económica” (Bustamante 1996: 205).

En América Latina y en los países del "Tercer Mundo", las expectativas frente las MFCS desde el principio fueron más amplias que en los países desarrollados. Se argumentaba que la seguridad de esta región dependía, ante todo, de los factores sociales y económicos y, en menor grado, de los militares. Según Isaac Caro - "Entre los principales objetivos de las MCM, en contexto latinoamericano, estaban la reducción de los presupuestos militares y de las importaciones de armas, de manera de contar con mayores recursos para el desarrollo social y económico de los países respectivos” (Caro 1994: 41). 
No obstante, en realidad, mientras que en la OSCE, después de la caída de la URSS, el enfoque fue replanteado de la prevención del conflicto militar a la necesidad de consolidación y estabilización de las relaciones pacíficas entre los Estados por medio de, por ejemplo, la implementación de las consecutivas generaciones de las MFCS, en OEA, este proceso en las dos últimas décadas ha avanzado mucho más despacio. En consecuencia, aunque en América Latina, teóricamente, predomina el enfoque "extenso" de las MFCS, en realidad la mayoría de los investigadores las perciben en su dimensión puramente militar - MCM. ${ }^{7}$ Mark Bromley y Catalina Perdomo (2005) constatan que, en el caso de la OEA, todavía es demasiado temprano para hablar de las MFCS. En realidad, esta organización ha conseguido elaborar y convencer a una parte de los Estados miembros a implementar las MFCS del borde de la I y II generación europea (así llamadas medidas "blandas"), que se basan en los contactos voluntarios entre las fuerzas armadas, realizados mediante el intercambio del personal con propósitos instructivos, la participación en conferencias o maniobras militares conjuntas, y no un "firme", obligatorio, independiente y objetivo control del, por ejemplo, armamento ${ }^{8}$. Además, merece la pena prestar una especial atención a la cuestión del control de armas convencionales. A pesar de que la OEA incluyó esta cuestión en el ámbito de las MFCS, es algo que raramente se lleva a cabo. En el informe de la Junta Interamericana de Defensa (JID) para la Comisión de Seguridad Hemisférica del Consejo Permanente de la OEA (OEA 2006: 19) relativo a la implementación de las MFCS en el hemisferio occidental, se ha demostrado que, en 2005, en ninguno de los Estados que entregaron los informes nacionales sobre $\mathrm{MFCS}^{9}$ se implementó las medidas recomendadas por la OEA en lo que se refiere al control de armamentos convencionales (Briones, Álvarez 2008: 19). Aunque los países de América Latina entregan voluntariamente los informes al Registro de Armas Convencionales llevado por United Nations Office for Disarmament Affairs

7 Así las entiende el autor de este artículo.

8 Las medidas más frecuentemente utilizadas en 2009 son las siguientes: 1. Intercambio de personal civil y militar teniendo en cuenta la capacitación regular y avanzada; 2. Realizar reuniones de alto nivel con la participación de los ministerios de defensa y de relaciones exteriores, a nivel bilateral, subregional y regional con el fin de iniciar un diálogo franco y directo sobre la evaluación conjunta de varios aspectos de la defensa y la seguridad, e intercambiar ideas y puntos de vista sobre los objetivos de la política de defensa nacional, así como medios comunes para abordar problemas comunes en esta materia; 3. Ampliar en los institutos de formación diplomática, las academias militares, los centros de investigación y las universidades, los seminarios, cursos y estudios previstos en las Declaraciones de Santiago y San Salvador; 4. Realizar programas de visitas de defensa, donde los representantes de los Estados Miembros de la OEA participantes visiten las instalaciones de defensa y las escuelas militares; 5 . Intensificar la cooperación, dentro de la estructura de la OEA, en el combate contra el terrorismo, la interdicción de drogas, la prevención del tráfico ilegal de armas pequeñas y livianas, el combate de la piratería, la prevención del contrabando, la realización de operaciones de búsqueda y rescate y la protección de los recursos naturales y los bienes arqueológicos (OEA 2012: 22-25).

9 En 2005, los entregaron sólo: Argentina, Brasil, Chile, Colombia, Ecuador, el Salvador, Honduras y Nicaragua.
Medidas de Fomento de la Confianza y Seguridad en el ámbito de la Organización de Estados Americanos

Paweł Trefler 
(UNODA) y participan en los mecanismos de notificación de Gastos Militares de la ONU, pero no todos de ellos lo hacen de manera rigurosa y sistemática. La actitud de muchos países del hemisferio occidental hacia el control de armamentos se evidencia en el hecho de que el 1 de marzo de 2013, entre los 21 de los 34 activos países miembros de la OEA, los cuales firmaron la Convención Interamericana sobre Transparencia en las Adquisiciones de Armas Convencionales (CITAAC), adoptada por la Asamblea General de la OEA el 7 de junio de 1999, sólo 16 países la ratificaron - entre otros, Argentina, Brasil y Chile, pero ya no EE.UU., Colombia o Bolivia (OEA 2013). Decididamente mejor se presenta la cuestión de ratificación por parte de los países de América Latina de los tratados regionales y universales referentes a la proscripción de armas de destrucción en masa. Por ejemplo, el Tratado para la Proscripción de las Armas Nucleares en la América Latina y el Caribe (Tratado de Tlatelolco) del 14 de febrero de 1969 fue ratificado por 33 países de la región, incluido Argentina y Brasil, los cuales desarrollaban sus programas nucleares. ${ }^{10}$

No cabe duda que el régimen de MFCS hemisférico, a pesar de los esfuerzos de la OEA, en comparación con la OSCE, es mucho menos avanzado. La razón de tal situación parece ser, ante todo, la falta de confianza de algunos países de América Latina en esta organización y, en consecuencia, la falta de verdadero interés en algunas de sus iniciativas. En la opinión de muchos latinos, la OEA y la JID, ligada con ella institucionalmente desde 2006, no son nada más que las herramientas que sirven a la realización de los intereses geoestratégicos de EE.UU. y, en realidad, las MFCS promovidas por esta organización tienen el objetivo de facilitar a los americanos el control sobre las fuerzas armadas de los países de América Latina. Aparte de eso, los países miembros de la OEA tienen intereses políticos divergentes por causa de, entre otros, su ubicación geográfica, la extensión de su territorio, la situación demográfica, los problemas que enfrentan, el potencial de sus fuerzas armadas y armas disponibles y el grado de sumisión al poder civil, así como también sus lazos internacionales. La OEA agrupa, pues, países demasiado diferenciados para que pueda actuar con plena eficacia en favor de la construcción del régimen hemisférico de MFCS. Además, esta organización nunca ha sido un buen foro para la solución pacífica de conflictos internacionales. Los países de América Latina, desanimados por el hecho de que durante la guerra fría EEUU trataron a la OEA de manera instrumental, aprendieron a contar más con los mecanismos creados ad hoc por ellos mismos, como, por ejemplo, Grupo de Contadora ${ }^{11}$,

${ }^{10}$ Para que los países signatarios respeten sus disposiciones, vigila una entidad creada especialmente para este fin: el Organismo para la Proscripción de las Armas Nucleares en la América Latina y el Caribe (OPANAL). Véase más sobre el tema de la proliferación de armas nucleares, biológicas y químicas (Coutto 2013: 81-106).

11 En el contexto de las MFCS, vale la pena recordar que en América Latina el primer documento que obligaba a los países a utilizar las MCM concretas fue el Acta de Contadora para la paz y la cooperación en Centroamérica, firmada el 6 de junio de 1986 por los gobiernos de Costa Rica, el Salvador, Guatemala, Honduras y Nicaragua en Ciudad de Panamá. 
organizaciones subregionales y mediaciones llevadas a cabo por los prominentes líderes políticos latinoamericanos. Por otra parte, la OEA es útil como una organización que fija el rumbo de las actividades y proporciona algunas herramientas que pueden, pero no necesariamente tienen que ser utilizados en las relaciones bilaterales o el foro de las organizaciones subregionales.

Un otro aspecto que dificulta la construcción del régimen de MFCS en el marco de la OEA, lo señaló un diplomático peruano, José Bellina Acevedo. Según su opinión - "El hecho de que no exista un riesgo eminente de conflicto que englobe a toda la región sumado a que, de presentarse una confrontación bélica, ésta sería exclusivamente entre dos actores hacen que, de alguna forma, los Estados no se decidan a adoptar este tipo de medidas en forma generalizada, sino mas bien que estén sujetas a la discrecionalidad de los actores potencialmente involucrados en un enfrentamiento" (Bellina Acevedo 2010: 26).

En lo que se refiere a la confianza mutua, los países de América Latina se basan más bien en la cooperación bilateral que en las soluciones elaboradas por la OEA. En consecuencia, entre los miembros de esta organización hay diferencias en el progreso de la implementación y evaluación de las MFCS. Actualmente, los regímenes de MFCS más avanzados funcionan en las relaciones entre Argentina y Chile y entre Argentina y Brasil. Vale la pena destacar que estos dos pares de países pertenecían hace más de 30 años a los más conflictivos en toda América del Sur, pero en los años 80 y al principio de los años 90 consiguieron acabar con la peligrosa rivalidad nuclear, territorial y por los recursos naturales, comenzando así el proceso de la integración. En el polo opuesto se encuentra la región andina, junto con Guyana, donde la situación internacional es inestable actualmente. Entre los países de esta región, todavía hay demasiados conflictos irresueltos, lo que dificulta considerablemente la construcción de la confianza entre ellos.

No hay que menospreciar las iniciativas de la OEA en el ámbito de las MFCS, no obstante, como lo ha advertido con razón Marcela Donadio (2010: 41), "estas medidas han sido difíciles de implementar, y los informes nacionales (sobre MFCS) no se caracterizan ni por su regularidad ni por su accesibilidad". ${ }^{2}$ Además, como ha señalado Ricardo Mario Rodríguez - "Son acciones elementales, de buena voluntad, que sin duda constituyen un primer paso positivo en el proceso de generar y aumentar la confianza entre los países. Sin embargo, su carácter voluntario, poco formal, y la ausencia de mecanismo de supervisión que garanticen su cumplimiento las hacen insuficientes" (Rodríguez 1996: 94). En realidad, de igual manera se presentan las MFCS establecidas en las relaciones bilaterales y subregionales, con la excepción de

${ }^{12}$ Los países miembros de la OEA y la JID deberían presentar anualmente los informes sobre la implementación de las MFCS. Hay países que lo hacen con regularidad: Chile, Brasil, Argentina, el Salvador y Guatemala; con menos regularidad: Canadá, Honduras, Nicaragua; pero también hay los que prácticamente no lo hacen: Bolivia, Guyana, Trinidad y Tobago, Venezuela (OEA 2012: 18-19)
Medidas de Fomento de la Confianza y Seguridad en el ámbito de la Organización de Estados Americanos

Paweł Trefler 
las anteriormente mencionadas relaciones argentino-chilenas, argentino-brasileñas y las soluciones elaboradas en América Central, las cuales se pueden encontrar en el Tratado Marco para la Seguridad Democrática del 15 de diciembre de 1995.

A pesar de que R. M. Rodríguez formuló su opinión sobre la condición de las MFCS hace varios años, desde aquel tiempo no se ha notado mucho progreso en cuanto a esta cuestión en el foro de la OEA. Aún falta mucho para que se realicen las recomendaciones que F. Rojas Aravena formuló acerca de las MFCS. Según él, para que sean eficaces, las MFCS deben ser: a) transparentes y abiertas; b) predecibles; c) recíprocas y equivalentes; d) destinadas a mejorar las percepciones; e) permanentes en tiempo; f) realistas en su ejecución; g) coherentes; h) verificables; i) deben contar con una fuerte legitimidad doméstica; j) variables según el número de actores (Rojas Aravena 1996: 47-49).

\section{Conclusiones}

Argentina, Brasil y Chile están predestinados para asumir de la OEA la responsabilidad principal por la construcción, implementación y evaluación de las MFCS en América Latina. Hay varios factores que lo determinan: 1. Disponen de las más grandes y profesionales fuerzas armadas sometidas al control civil; 2. Son - tomando en cuenta la realidad de América del Sur - fuertes institucionalmente y estables políticamente; 3. Se involucran activamente en las iniciativas internacionales en favor de consolidar la democracia y establecer América del Sur como una zona de paz; ${ }^{13} 4$. Tienen bastante experiencia en la resolución pacífica de conflictos; 5 . Promueven y actúan activamente en favor de la integración multifacética de América del Sur (vide: Mercado Común del Sur - MERCOSUR); 6. Chile y Argentina fueron los primeros propagadores de las MFCS en América Latina ${ }^{14}$ y hoy, en las relaciones bilaterales, disponen de la más amplia gama de las MFCS; 7. Argentina, Brasil y Chile han institucionalizado la cooperación bilateral estratégica en favor de la seguridad y defensa (Véase más: Flemes 2004); ${ }^{15}$ 8. Chile en 1997 y Argentina en 1999, como

${ }_{13}$ El 27 de julio de 2002, durante la II Cumbre Suramericana en Guayaquil (Ecuador), el MERCOSUR y la Comunidad Andina (CAN) crearon la Zona de Paz Sudamericana, a la que accedieron también Venezuela, Guyana y Surinam.

14 Fue la iniciativa de Santiago de Chile, apoyada por Buenos Aires, la que dio impulso a la organización en 1995 de la Conferencia Regional sobre MFCS.

${ }_{15}$ Argentina colabora con Chile en el marco del Comité Permanente de Seguridad (COMPERSEG), Reuniones de Consulta entre Ministros de Relaciones Exteriores y de Defensa, y con Brasil dentro del marco del Grupo de Trabajo bilateral de Defensa Argentino-Brasileño, Mecanismo de Consulta y Coordinación en Brasil y Argentina en materia de Defensa y Seguridad Internacional (MCC), Mecanismo Permanente de Análisis Estratégico (MAE) y Mecanismo de Diálogo Político Estratégico de nivel Viceministerial (MDPEVM). Chile y Brasil mantienen contactos regulares en el marco del Grupo de Trabajo Bilateral de Defensa Chileno-Brasileño (GTBD). 
primeros países en América Latina, publicaron Libros Blancos de Defensa; 9. Argentina, Brasil y Chile publican con regularidad documentos referentes a su política de seguridad y defensa, 10. Estos países, entre pocos en América Latina, desde el principio proporcionan a JID/OEA y UNODA informes regulares y confiables referentes a las MFCS; 11 . Argentina y Chile han elaborado la Metodología estandarizada común para la medición de los Gastos de Defensa, la cual se ha convertido en el modelo para otros países de la región; 12. Juntos, estos países pueden constituir un contrapeso real a las influencias políticas, económicas y militares de EEUU en América Latina.

Argentina, Brasil y Chile, después del 11 de septiembre de 2001, cuando, por razones obvias, disminuyó el interés de EEUU por América Latina, empezaron a cumplir con las expectativas depositadas en ellos. Brasil empezó a desempeñar el papel principal. En respuesta a la crisis colombo-venezolana, la cual se iba agudizando desde 2003, el 18 de diciembre de 2004 llevó a cabo la creación de la UNASUR, ${ }^{16}$ y el 16 de diciembre de 2008, en la "super-cumbre" latinoamericana ${ }^{17}$ en la ciudad brasileña de Costa do Sauípe, el Consejo de Defensa Suramericano (CDS). Los objetivos fijados para este órgano, el cual actúa bajo los auspicios de la UNASUR, son bastante ambiciosos. Tiene que asumir el papel de un mecanismo institucionalizado para la prevención y solución de conflictos por vía diplomática, así como también ser el foro de diálogo entre los ministerios de defensa de los países miembros. Además, debe ocuparse de la construcción de las MFCS y del reforzamiento del control civil sobre las fuerzas armadas (Serbin 2009: 150-151). UNASUR ya ha asumido de la OEA una gran parte de responsabilidad por la creación de las MFCS en América del Sur, pero la presentación de las acciones propuestas y realizadas por ella sobrepasa el marco de este artículo.

Actualmente, los países de América Latina tienen que intentar implementar plenamente las MFCS "obligatorias (aunque a priori voluntarias), multilaterales, significativas desde el punto de vista militar, con mecanismos de verificación" (Sibilla 2009: 19). En este contexto, muy importante es la institucionalización de las MFCS, las cuales - según M. Bromley i C. Perdomo (2005) - "En la mayoría de los países latinoamericanos se acuerdan en reuniones presidenciales que implican compromisos personales entendidos como «políticas gubernamentales» en lugar de "políticas estatales»". El proceso de la institucionalización de las MFCS debería ser acompañado por su continua actualización, además de la uniformización y mayor frecuencia de presentación por parte de los Estados de informes sobre la implementación de las MFCS,

${ }^{16}$ Originalmente bajo el nombre Comunidad de Naciones Suramericanas.

17 En el mismo tiempo y lugar hubo cuatro cumbres (Grupo de Río, MERCOSUR, UNASUR y de los países de América Latina y el Caribe - CALC). En total, en las cumbres participaron los representantes de 33 países americanos, con la excepción de los líderes de los Estados Unidos, Canadá, Colombia y Perú. Aparte de las organizaciones mencionadas más arriba, en la "super-cumbre" fueron representados otros bloques de integración, tales como CAN, Alianza Bolivariana para los Pueblos de Nuestra América ALBA, Caribbean Community and Common Market (CARICOM) y el Sistema de la Integración Centroamericana (SICA).
Medidas de Fomento de la Confianza y Seguridad en el ámbito de la Organización de Estados Americanos

Paweł Trefler 
insistiendo, además, en su veracidad. Hay que limitar también la autonomía de las fuerzas armadas en cuanto a la definición y realización de la política de defensa y, paralelamente, reforzar el sector civil en el ámbito de defensa e informar más ampliamente a la opinión pública sobre las cuestiones de defensa. Aparte de eso, no hay que olvidarse de la necesidad de reforzar el control de armamentos convencionales y gastos militares y también emprender la tarea de limitarlos. Además, vale la pena intentar desarrollar las MFCS relacionadas a las nuevas amenazas para frenar la transferencia de la inestabilidad.

\section{Bibliografía}

Arévalo de León B. (2002), Good Governance in Security Sector as Confidence Building Measure in the Americas: toward Pax Democratica, "DCAF Conference Papers", Geneva, disponible en: http://disarm.igc.org/2009backup/Regional/Arevalo.pdf, consulta: 10.08.2013.

Bellina Acevedo J. (2010), Medidas de Fomento de la Confianza: la realidad regional, en: Confianza y Seguridad en América del Sur, Consejo de Defensa Suramericano UNASUR, "Cuadernos de Defensa", no. 2, octubre, disponible en: http://www.opalc.org/web/images/ stories/INTE/Confianza_y_seguridad_en_America_del_Sur1.pdf, consulta: 10.10.2013.

Briones S. R., Álvarez R. V. (2008), ¿Construyendo confianzas? Fronteras, FFAA y Política en América Latina, Santiago de Chile, disponible en: http://issuu.com/flacso.chile/docs/ rss_2008_1, consulta: 10.08.2013.

Bromley M., Perdomo C. (2005), CBMen América Latina y el efecto de la adquisición de armas por parte de Venezuela, Real Instituto Elcano de Estudios Internacionales y Estratégicos, "Documento de Trabajo", no 41, disponible en: http://www.realinstitutoelcano.org/ documentos/219/219_BromleyPerdomo.pdf, consulta: 10.08.2013.

Bustamante F. (1996), La cuestión de las medidas de confianza mutua en el contexto de la sub-región andina, en: Balance estratégico y medidas de confianza mutua, F. Rojas Aravena (ed.), Santiago de Chile.

Caro I. (1994), Medidas de confianza mutua en Sudamérica, "Fuerzas Armadas y Sociedad", vol. 9, no 2, abril - junio, disponible en: http://www.fasoc.cl/files/articulo/ ART411a321c666ee.pdf, consulta: 10.08.2013.

Caro I. (1994), Presentación, en: Medidas de confianza mutua en América Latina, A. Varas, I. Caro (ed.), Santiago de Chile.

Child J. (1994), Medidas de confianza mutua en América Central, en: Medidas de confianza mutua en América Latina, A. Varas, I. Caro (ed.), Santiago de Chile.

Donadio M. (2010), El Consejo de Defensa Suramericano y su papel en el desarrollo de confianza mutua, en: Confianza y Seguridad en América del Sur, Consejo de Defensa Suramericano UNASUR, "Cuadernos de Defensa", no. 2, octubre, disponible en: http://www. opalc.org/web/images/stories/INTE/Confianza_y_seguridad_en_America_del_Sur1.pdf, consulta: 10.10 .2013 .

Holum J. (1996), El avance de la confianza en el hemisferio, en: Balance estratégico y medidas de confianza mutua, F. Rojas Aravena (ed.), Santiago de Chile.

Fuentes C, Millet P. (1997), Chile, Bolivia Perú: los nuevos desafíos de la integración, Santiago de Chile. 
Jácome F. (2008), Venezuela en el contexto de las Medidas de Fomento de la Confianza y la Seguridad, Instituto Latinoamericano de Investigaciones Sociales, disponible en: http://invesp.org.ve/wp-content/uploads/2013/03/DocumentoFrancineJacomeFinal.pdf, consulta: 10.08.2013.

Lachowski Z. (2004), Confidence - And Security - Building Measures in the New Europe, "SIPRI Research Report", no 18, New York.

OEA (2003), Consenso de Miami, 04.02.2003, disponible en: http://www.oas.org/csh/ spanish/documentos/re00218s04.doc, consulta: 8.08.2013.

OEA (2006), Actualización del inventario de las MFCS, JID, 27.11.2006, disponible en: http://www.oas.org/csh/spanish/Foro.asp, consulta: 9.08.2013.

OEA (2009), Lista consolidada de MFCS que se deben notificar de conformidad con las resoluciones de la OEA, 15.01.2009, disponible en: http://www.oas.org/csh/spanish/mfclist. asp, consulta: 9.08.2013.

OEA (2012), Inventario de MFCS 2010, OEA/JID, marzo 2012, disponible en: http://scm. oas.org/pdfs/2012/CP28614P.pdf, consulta: 10.08.2013.

OEA (2013), Estado de las ratificaciones a la Convención Interamericana sobre Transparencia en las Adquisiciones de Armas Convencionales (CITAAC), 01.03.2013, disponible en: http:// scm.oas.org/doc_public/SPANISH/HIST_13/CP30272S08.doc, consulta: 9.08.2013.

Riquelme Rivera J. (2011), El Mercosur y la formación de una comunidad pluralista de seguridad en el Cono Sur, "Revista Enfoques", vol. IX, no 14, disponible en: http://www. politicaygobierno.cl/wp-content/uploads/2011/07/Enf14_Riquelme.pdf, $\quad$ consulta: 10.08.2013.

Rodríguez R. M. (1996), El impacto de la revitalización del sistema interamericano en la seguridad regional, en: Balance estratégico y medidas de confianza mutua, F. Rojas Aravena (ed.), Santiago de Chile.

Rojas Aravena F. (1996), Balance estratégico y medidas de confianza mutua, Santiago de Chile.

Rojas Aravena F. (1996), Medidas de confianza mutua y balance estratégico: un vínculo hacia la distensión y la estabilidad, en: Balance estratégico y medidas de confianza mutua, F. Rojas Aravena (ed.), Santiago de Chile.

Serbin A. (2009), América del Sur en un mundo multipolar: ¿es la Unasur la alternativa?, "Nueva Sociedad", no. 219, enero-febrero, disponible en: http://www.nuso.org/ upload/ articulos/3588_1.pdf, consulta: 10.08.2013.

Sibilla G. (2009), Hacia el Registro Suramericano de Gastos de Defensa: Construyendo Confianza, Materia Prima de la Integración, Centro de Estudios Estratégicos para la Defensa "Manuel Belgrano", "Revista de la Defensa" no. 4, disponible en: http://issuu.com/ceepade/ docs/rd4, consulta: 11.08.2013.

Tulliu S., Schmalberger T. (2003), En buenos términos con la seguridad: diccionario sobre control de armamentos, desarme y fomento de la confianza, Ginebra.

Varas A., Caro I. (1994), (eds.), Medidas de confianza mutua en América Latina, Santiago de Chile.

Zięba R. (2008), (ed.), Bezpieczeństwo międzynarodowe po zimnej wojnie, Warszawa.
Medidas de Fomento de la Confianza y Seguridad en el ámbito de la Organización de Estados Americanos

Paweł Trefler 